\title{
Dysregulation of Circular Antigen-Specific T Cells Anergy in Autoimmune Vitiligo
}

\author{
Atsushi Tanemura*, Lingli Yang, Ichiro Katayama \\ Department of Dermatology Integrated Medicine, Osaka University Graduate School of Medicine, Osaka, Japan \\ Email: *tanemura@derma.med.osaka-u.ac.jp
}

How to cite this paper: Tanemura, A., Yang, L. and Katayama, I. (2018) Dysregulation of Circular Antigen-Specific T Cells Anergy in Autoimmune Vitiligo. Journal of Cosmetics, Dermatological Sciences and Applications, 8, 133-139.

https://doi.org/10.4236/jcdsa.2018.83015

Received: August 8, 2018

Accepted: September 17, 2018

Published: September 20, 2018

Copyright () 2018 by authors and Scientific Research Publishing Inc. This work is licensed under the Creative Commons Attribution International License (CC BY 4.0).

http://creativecommons.org/licenses/by/4.0/

(c) (i) Open Access

\begin{abstract}
Background: Vitiligo is an autoimmune disorder related to melanocyte loss; however, the exact interplay between antigen-specific autoimmunity and local oxidative stress remains unclear. Recently, the migration ability and number of Foxp3-expressing regulatory $\mathrm{T}$ cells (Tregs) in lesional skin was found to be reduced in vitiligo patients. Objectives: We aimed to clarify the T cell anergy status of melanocytes by focusing on the impaired equivalence of peripheral melanocyte-specific cytotoxic $\mathrm{T}$ cells and functional Tregs in patients with progressive vitiligo. Materials and methods: Ten progressive vitiligo patients and 10 age-matched healthy individuals were enrolled in this study. We analyzed the number of functional Tregs in progressive vitiligo patients and compared the findings with those of controls. Next, to assess the suppressive activity of Tregs on melanocyte-specific $\mathrm{T}$ lymphocytes, we strictly purified the functional Tregs fraction and Melan-A-specific $\mathrm{CD}^{+} \mathrm{T}$ cells and co-cultured these cells with each other. The number of Melan-A-specific $\mathrm{CD} 8^{+} \mathrm{T}$ cells was then counted by FACS. In addition, the expression of the representative exhaustion markers PD-1 and CTLA-4 on functional Tregs was assessed in vitiligo patients and normal controls. Results: The number of functional Tregs itself was not significantly decreased in the blood of vitiligo patients compared to healthy controls. However, the cytotoxic T cell (CTL) proliferation was significantly decreased after cultivation with Tregs from healthy individuals $(\mathrm{p}<0.01)$, and this decrease in CTLs was less marked after cultivation with Tregs from vitiligo patients. Conclusions: We demonstrated a reduced suppressive function of activated Tregs on Melan-A-specific CTLs in the circulating cells of vitiligo patients compared with healthy controls. This result suggests that $\mathrm{T}$ cell anergy with Tregs dysfunction may participate in the immune response to melanocytes in vitiligo patients.
\end{abstract}

\section{Keywords}

Autoimmune Vitiligo, T Cell Anergy, Functional Regulatory T Cell 


\section{Introduction}

Vitiligo is an autoimmune disorder related to melanocyte loss; however, the exact interplay between antigen-specific autoimmunity and local oxidative stress remains unclear [1]. Recently, melanocyte-specific $\mathrm{T}$ lymphocytes with naive characteristics were observed in HLA-A2 melanoma patients and healthy donors [1]. In addition, a high frequency of these cells that might influence melanocyte disappearance was also detected in cases of progressive vitiligo. Foxp3-expressing regulatory $\mathrm{T}$ cells (Tregs) are the main player involved in the suppression of the aberrant immune response against self-antigens. Recently, the migration ability and number of Tregs in lesional skin were found to be reduced in vitiligo patients [2] [3]. We previously detected and described self-reactive $\mathrm{T}$ cells with an anergic phenotype, which is immunologically tolerant, in Tregs [4]. In this study, we aimed to clarify the $\mathrm{T}$ cell anergy status regarding melanocyte-specific antigen by focusing on the impaired equivalence of peripheral melanocyte-specific cytotoxic $\mathrm{T}$ cells and functional Tregs in patients with progressive vitiligo.

\section{Materials and Methods}

Ten progressive vitiligo patients and 10 age-matched healthy individuals who gave their written informed consent were enrolled in this study, and the details of the patients' demographic findings are shown in Table 1. We enrolled progressive vitiligo patients who visited our department from 2015 to 2017 and showed no physical problems based on the findings of blood tests. Ten healthy individuals without any remarkable history or medication participated in this study as healthy controls. This study was approved by the ethics committee of our university. Blood collection was performed before steroid half pulse therapy in cases \#3 and 10 in order to eliminate the suppressive effect of systemic steroid on immunity. We properly isolated a functional subset of human Tregs that maintained their suppressive activity according to a previous publication [5] and purified $\mathrm{CD}^{+} \mathrm{T}$ cells and antigen-presenting cells (APC) simultaneously from the peripheral blood mononuclear cells (PBMCs) of vitiligo patients $(\mathrm{n}=10)$ and healthy controls $(\mathrm{n}=3)$. $\mathrm{CD} 8^{+} \mathrm{T}$ cells were co-cultured with APCs stimulated with HLA-A0201-restricted Melan-A peptides and with or without Tregs for 10 days.

\section{Results}

1) The number of Melan-A-specific cytotoxic T lymphocytes (CTLs) was significantly increased in the blood of vitiligo patients compared to that of healthy controls (Figure 1(a)). Several previous reports showing reduced Treg homing into the skin of vitiligo patients analyzed all fractions of Tregs, including not only functional but also nonfunctional populations, in humans

[2] [3]. Defects in the skin-homing chemokine CCL22 may play a role in the paucity of Tregs in vitiligo skin [2] [6]. To evaluate the changes in the num- 
bers of functional Tregs, we isolated and examined only the functional Treg fraction with a high-Foxp3 and low-CD45RO expression in CD4 $4^{+}$cells. The number of functional Tregs itself was not significantly decreased in the blood of vitiligo patients compared to that of healthy controls (Figure 1(b)).

2) Next, to explore whether or not the suppressive function of Tregs was disturbed in the PBMCs of vitiligo patients, we co-cultured Melan-A-specific CTLs with or without $\mathrm{CD} 4^{+} \mathrm{CD} 25^{+} \mathrm{T}$ cells and compared the cell number and proliferation between the two conditions (Figure 1(c)). While CTL proliferation was significantly decreased after cultivation with Tregs from healthy individuals (Figure 1(c), left 2 lanes in the lower panel), the decrease in the number of CTLs was less marked after cultivation with Tregs from vitiligo patients (Figure 1(c), right 2 lanes in the lower panel).

3) The proportion of PD-1 has been reported to be decreased in Tregs of active vitiligo patients, suggesting its role in Treg exhaustion. We examined the expression if CTLA-4 and PD-1 in CTLs, and not Tregs, in order to analyze the exhaustion status in the direct $\mathrm{T}$ cell response to melanocytes in vitro. We found a weakened increase in the CTLA-4 expression of CTLs after cultivation in vitiligo patients compared to healthy controls (Figure 1(d)). The CTLA-4 expression may be less influenced by Tregs in patients with vitiligo than in healthy controls.

\section{Discussion}

Autoimmunity to mature melanocytes and their precursors is known to affect the onset and maintenance of vitiligo. The autoimmune response is reported to involve several sets of $\mathrm{T}$ cells, including Tregs and Th17 cells [7] [8]. In the

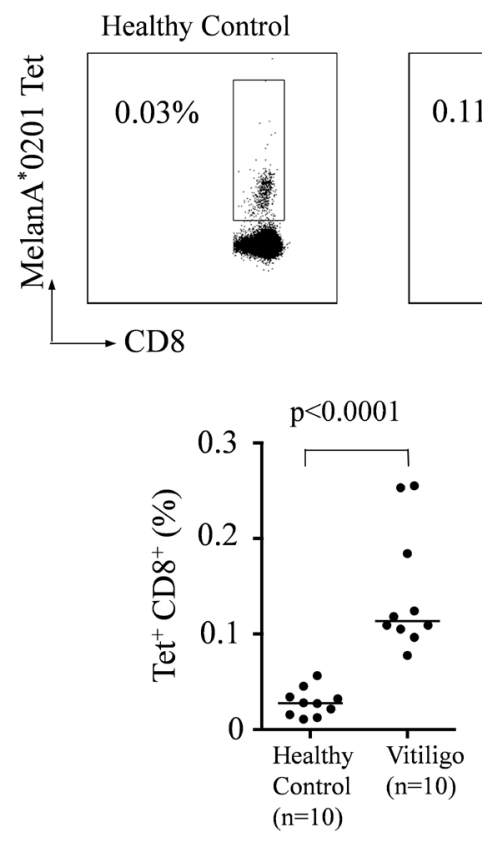

(a)

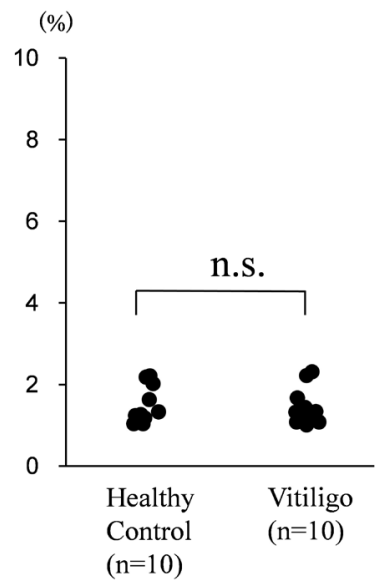

(b) 


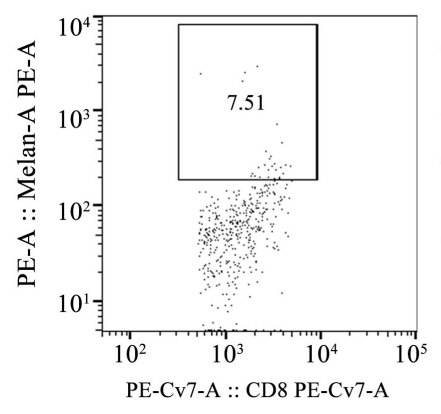

(w/o Treg)

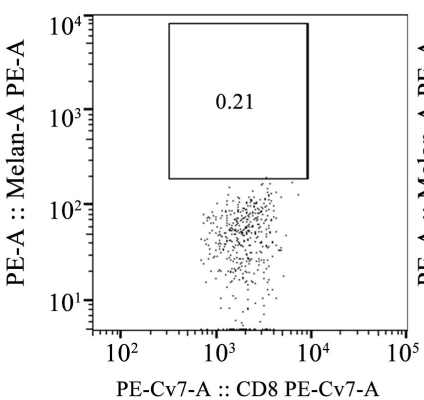

(with Treg)

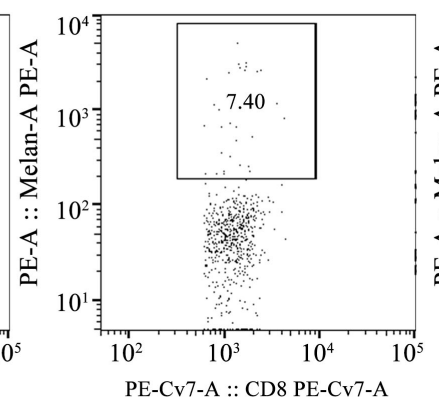

(w/o Treg)

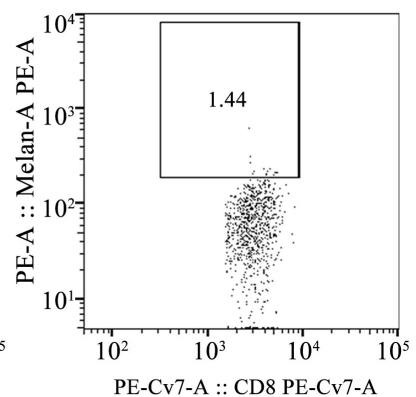

(with Treg)

Helathy Control

Vitiligo

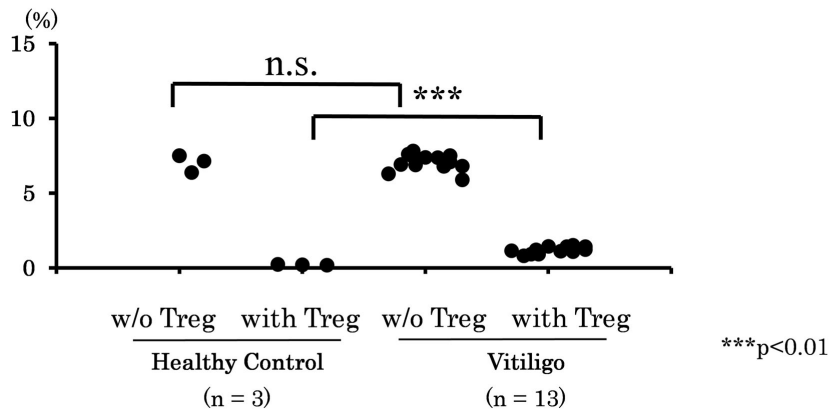

(c)
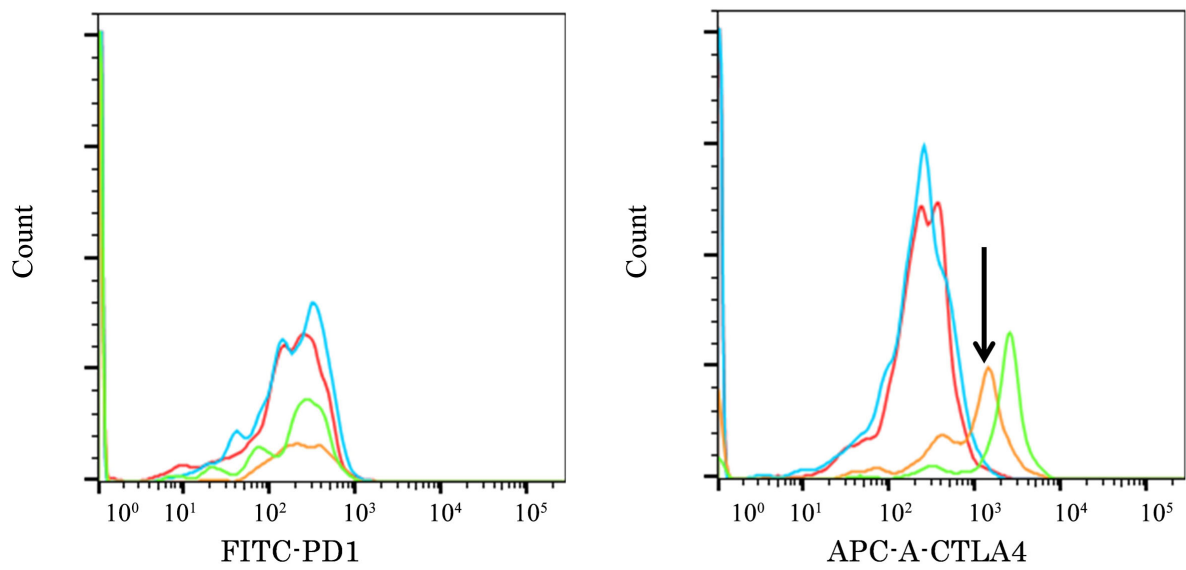

Healthy Control

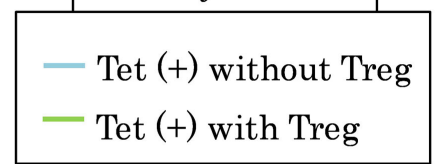

(d)

Figure 1. (a) The frequency of Melan-A-specific CTL was higher in vitiligo patients than in healthy controls. The frequency of HLA-A ${ }^{\star} 0201$-restricted Melan-A-specific CTL $\left(\mathrm{Tet}^{+} \mathrm{CD}^{+}\right.$cell) was $0.03 \%$ in healthy controls and $0.11 \%$ in vitiligo patients. The number of $\mathrm{Tet}^{+} \mathrm{CD}^{+}$cells was significantly greater in vitiligo patients than in healthy controls $(\mathrm{p}<0.0001)$; (b) The number of functional Tregs was not markedly different between vitiligo patients and healthy controls. The figure shows the representative data of fractionated Tregs in the circulation of a healthy control (left panel) and a vitiligo patient (right panel). No significant difference was noted in the number of functional Tregs highly positive for FoxP3 and negative for CD45 RA; (c) The suppressive function of functional Tregs was weaker in vitiligo patients than in healthy controls. The number of $\mathrm{Tet}^{+} \mathrm{CD}^{+}$cells was decreased on co-culture with $\mathrm{CD} 4{ }^{+} \mathrm{CD} 25^{+}$cells and was significantly lower after culture with $\mathrm{CD} 4^{+} \mathrm{CD} 25^{+}$cells from vitiligo patients than after culture with those from healthy controls ( $\mathrm{p}<0.01$, lower panel); (d) A Representative FACS analysis of the exhaustion markers in CTLs. The CTLA-4 (right panel) expression in Melan-A-specific CTLs not PD-1 (left panel) was deceased after co-culture with functional Tregs. The arrow indicates relatively decreased CTLA-4 expression in vitiligo patients compared to normal individuals. 
Table 1. Demographics of vitiligo patients enrolled in this study.

\begin{tabular}{|c|c|c|c|c|}
\hline Patient & Age & Gender & $\begin{array}{l}\text { Disease duration at the time of } \\
\text { analysis (yrs) }\end{array}$ & Treatments \\
\hline 1 & 67 & $\mathrm{~F}$ & 1 & Ointment, phototherapy \\
\hline 2 & 24 & M & 5 & Ointment, phototherapy \\
\hline 3 & 72 & M & 10 & $\begin{array}{l}\text { Ointment, phototherapy, } \\
\text { systemic steroid }\end{array}$ \\
\hline 4 & 41 & M & 10 & Ointment, phototherapy \\
\hline 5 & 24 & $\mathrm{~F}$ & 5 & Ointment, phototherapy \\
\hline 6 & 62 & M & 21 & Ointment, phototherapy \\
\hline 7 & 73 & M & 26 & None \\
\hline 8 & 27 & $\mathrm{~F}$ & 3 & Ointment, phototherapy \\
\hline 9 & 56 & M & 7 months & Ointment, phototherapy \\
\hline 10 & 63 & $\mathrm{~F}$ & 2 & $\begin{array}{l}\text { Ointment, phototherapy, } \\
\text { systemic steroid }\end{array}$ \\
\hline
\end{tabular}

present study, we investigated whether or not functional Tregs extracted from vitiligo patients truly had a degraded function of melanocyte-specific $\mathrm{T}$ cells. We were able to prove the disturbance of the suppression function by analyses of peripheral blood and proposed a mechanism underlying the autoimmunity to melanocytes in vitiligo. Thus far, it has not been proven that Tregs infiltrating the vitiligo skin truly exert a suppressive function to melanocyte-specific CTLs. We showed that the Treg activity was insufficient to suppress antigen-specific $\mathrm{T}$ cells in a small number of vitiligo patients, suggesting that a larger sample size is needed for future investigations. The suppressive function of Tregs is partly mediated through the programmed death-1 (PD-1)/PD-L1 pathway. Although the $\mathrm{PD}-1$ expression on the surface of Tregs has been reported to be increased [9], we observed no marked changes in the PD-1 expression. The CTLA-4 expression but not the PD-1 expression was decreased after co-cultivation with functional Tregs in representative patients (Figure 1(d)). This may have caused the difference of the Tregs' status and analyzed vitiligo population between the present study and previous report. Although the functional impairment in Tregs was found to be correlated with disease progression [10] [11], this is the first report to directly demonstrate that the suppressive function of activated Tregs on Melan-A-specific CTLs was significantly higher in vitiligo patients than in healthy controls. Our findings also suggest the functional attenuation of Tregs based on an examination of precisely isolated cell culture and the expression of exhaustion markers. To confirm our results, further investigations of the exhaustion marker expression and the mutual interplay of these markers in Tregs will be required in order to evaluate $\mathrm{T}$ cell anergy in vitiligo patients. 


\section{Acknowledgements}

We thank Kenju Nishida, Eriko Nobuyoshi for their expert technical assistance.

\section{Funding}

This work was supported by the Japanese Grant-in-aid for Scientific Research.

\section{Grant Support}

This work was supported in part by a grant from the Ministry of Education, Culture, Sports, Science and Technology of Japan and a grant from the Ministry of Health, Labour and Welfare.

\section{Conflicts of Interest}

The authors have no conflicts of interest to declare.

\section{References}

[1] Laddha, N.C., Dwivedi, M., Mansuri, M.S., Gani, A.R., Ansarullah, M., Ramachandran, A.V., et al. (2013) Vitiligo: Interplay between Oxidative Stress and Immune System. Experimental Dermatology, 22, 245-250. https://doi.org/10.1111/exd.12103

[2] Klarquist, J., Denman, C.J., Hernandez, C., Wainwright, D.J., Strickland, F.M., Overbeck, A., et al. (2010) Reduced Skin Homing by Functional Treg in Vitiligo. Pigment Cell \& Melanoma Research, 23, 276-286. https://doi.org/10.1111/j.1755-148X.2010.00688.x

[3] Abdallah, M., Lofti, R., Othman, W. and Galal, R. (2014) Assessment of Tissue $\mathrm{FoxP}^{+}, \mathrm{CD}^{+}$and $\mathrm{CD}^{+}{ }^{+} \mathrm{T}$-Cells in Active and Stable Nonsegmental Vitiligo. International Journal of Dermatology, 53, 940-946. https://doi.org/10.1111/ijd.12160

[4] Maeda, Y., Nishikawa, H., Sugiyama, D., Ha, D., Hamaguchi, M., et al. (2014) Detection of Self-Reactive CD8 ${ }^{+} \mathrm{T}$ Cells with an Anergic Phenotype in Healthy Individuals. Science, 346, 1536-1540. https://doi.org/10.1126/science.aaa1292

[5] Miyara, M., Yoshioka, Y., Kitoh, A., Shima, T., Wing, K., Niwa, A., et al. (2009) Functional Delineation and Differentiation Dynamics of Human CD4 ${ }^{+} \mathrm{T}$ Cells Expressing the FoxP3 Transcription Factor. Immunity, 30, 899-911. https://doi.org/10.1016/j.immuni.2009.03.019

[6] Eby, J.M., Kang, H.K., Tully, S.T., Bindeman, W.E., Peiffer, D.S., et al. (2015) CCL22 to Activate Treg Migration and Suppress Depigmentation in Vitiligo. Journal of Investigative Dermatology, 135, 1574-1580. https://doi.org/10.1038/jid.2015.26

[7] Hegazy, R.A., Fawzy, M.M., Gawdat, H.I., Samir, N. and Rashed, L.A. (2014) T Helper 17 and Tregs: A Novel Proposed Mechanism for NB-UVB in Vitiligo. Experimental Dermatology, 23, 283-286. https://doi.org/10.1111/exd.12369

[8] van Geel, N.A., Mollet, I.G., Schepper, S.D., Tjin, E.P.M., Vermaelen, K., Clark, R.A., et al. (2010) First Histopathological and Immunophenotypic Analysis of Early Dynamic Events in a Patient with Segmental Vitiligo Associated with Halo Nevi. Pigment Cell \& Melanoma Research, 23, 375-384. https://doi.org/10.1111/j.1755-148X.2010.00703.x

[9] Tembhre, M.K., Parihar, A.S., Sharma, V.K., Sharma, A., Chattopadhyay, P. and Gupta, S. (2015) Alteration in Regulatory T Cells and Programmed Cell Death 1-Expressing Regulatory T Cells in Active Generalized Vitiligo and Their Clinical 
Correlation. British Journal of Dermatology, 172, 940-950.

https://doi.org/10.1111/bjd.13511

[10] Lili, Y., Yi, W., Ji, Y., Yue, S., Weimin, S. and Ming, L. (2013) Global Activation of $\mathrm{CD}^{+}$Cytotoxic $\mathrm{T}$ Lymphocytes Correlates with an Impairment in Regulatory $\mathrm{T}$ Cells in Patients with Generalized Vitiligo. PLoS One, 7, e37513. https://doi.org/10.1371/journal.pone.0037513

[11] Ahmed, M.B., Zaraa, I., Rekik, R., Elbeldi-Ferchiou, A., Kourda, N., Belhadj, H.M., et al. (2012) Functional Defects of Peripheral Regulatory T Lymphocytes in Patients with Progressive Vitiligo. Pigment Cell \& Melanoma Research, 25, 99-109.

https://doi.org/10.1111/j.1755-148X.2011.00920.x

\section{Abbreviations}

Treg: regulatory T cell, APC: antigen presenting cell, CTLs: cytotoxic T lymphocytes, CTLA-4: cytotoxic T-lymphocyte antigen 4, PD-1: programmed cell death 1. 